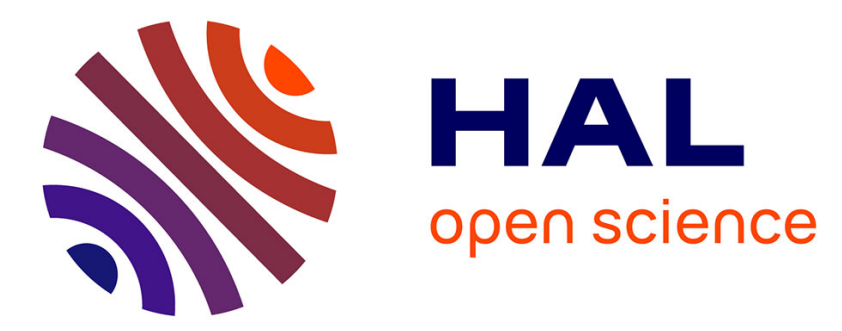

\title{
Optimal emission policy under the risk of irreversible pollution
}

\author{
Alain Ayong Le Kama, Aude Pommeret, Fabien Prieur
}

\section{To cite this version:}

Alain Ayong Le Kama, Aude Pommeret, Fabien Prieur. Optimal emission policy under the risk of irreversible pollution. Journal of Public Economic Theory, 2014, 16 (6), pp.959-980. 10.1111/jpet.12085 . hal-01280775

\section{HAL Id: hal-01280775 \\ https://hal.univ-grenoble-alpes.fr/hal-01280775}

Submitted on 4 Mar 2016

HAL is a multi-disciplinary open access archive for the deposit and dissemination of scientific research documents, whether they are published or not. The documents may come from teaching and research institutions in France or abroad, or from public or private research centers.
L'archive ouverte pluridisciplinaire HAL, est destinée au dépôt et à la diffusion de documents scientifiques de niveau recherche, publiés ou non, émanant des établissements d'enseignement et de recherche français ou étrangers, des laboratoires publics ou privés. 


\title{
Optimal emission policy under the risk of irreversible pollution*
}

\author{
Alain Ayong Le Kama ${ }^{\dagger} \quad$ Aude Pommeret Fabien Prieur $^{\S}$
}

\begin{abstract}
We consider an optimal consumption and pollution problem that has two important features. Environmental damages due to economic activities may be irreversible and the level at which the degradation becomes irreversible is unknown. Particular attention is paid to the situation where agents are relatively impatient and/or do not care a lot about the environment and/or Nature regenerates at low rate. We show that the optimal policy of the uncertain problem drives the economy in the long run toward a steady state while, when ignoring irreversibility, the economy follows a balanced growth path accompanied by a perpetual decrease in environmental quality and consumption, both asymptotically converging toward zero. Therefore, accounting for the risk of irreversibility induces more conservative decisions regarding consumption and polluting emissions. In general, however, we cannot rule out situations where the economy will optimally follow an irreversible path and consequently, will also be left, in the long run, with an irreversibly degraded environment.
\end{abstract}

Keywords: Optimal control, irreversibility threshold, uncertainty, optimal reversible and irreversible policy.

JEL codes: D81, Q54, Q58

*This work has benefited from the support of the French National Research Agency, reference ANR-09BLAN-0350-01. Preliminary versions of the paper were presented to the conference "Managing Climate Change" at the Collège de France, Paris, to the $4^{\text {th }}$ "World Congress of Environmental and Resource Economists", Montreal, Canada, to the "Public Good, Public Project and Externality" sessions at the Annual Conference of the Association for Public Economic Theory (PET'10), Istanbul, Turkey and to the "Monte Verità Conference on Sustainable Resource Use and Economic Dynamics - SURED 2010", Ascona, Switzerland.

${ }^{\dagger}$ EQUIPPE, Université de Lille 1. Email: adayong@univ-paris1.fr

‡Université de Lausanne and IREGE, Université de Savoie. E-mail address: aude.pommeret@univsavoie.fr.

§INRA-LAMETA, Université Montpellier I, France. E-mail: prieur@supagro.inra.fr. 


\section{Introduction}

We focus on situations where economic activities may translate into an irreversible degradation of the environment. The notion of irreversibility conveys the idea that when natural systems are submitted to strong perturbations, typically pollution, they may be incapable of regenerating. On the one hand, it points out that the regeneration capacity of Nature may change in response to the evolution of pollutants' concentration. On the other, it emphasizes that natural regeneration may exhibit a threshold effect, meaning that the recovering process may be discontinuous and non monotonic with respect to pollution. Whenever the level of pollution is kept below the threshold, Nature is able to assimilate part of this stock. However, as soon as pollution exceeds this critical value, it causes the assimilation to cease. Because of the irreversibility of damages, ecosystems exhibit multiple equilibria. Multiplicity implies that ecosystems, when facing strong changes, are unable to recover their initial state. Rather, they may be caught in a new highly polluted equilibrium. Irreversible degradation of the environment is not innocuous to the economy since people are more and more concerned with Nature and the quality of the environment surrounding them. This raises the important question of how individuals should behave with respect to the environment, provided that they run (and affect) the risk of facing an irreversibly degraded environment.

Irreversibility is involved in local pollutant problems such as the eutrophication of lakes, the salinification of soils or the loss of biodiversity because of land use (Dasgupta and Mäler 2003). Besides, there is more and more evidence that global environmental threats, like global warming, are also associated with irreversible pollution. Indeed, experts of the second working group of the IPCC (2007) have identified positive climate feedbacks due to emissions of greenhouse gases (GHG). Consequences of increasing emission levels and concentrations of GHG on the regeneration capacity of natural ecosystems can be summarized as follows. Oceans, that form the most important carbon sink, display a buffering capacity that begins saturating. At the same time, the assimilation capacity of terrestrial ecosystems (lands, forests, the other important carbon sink) will likely peak by mid-century and then decline to become a net source of carbon by the end of the present century. Therefore, the problem is even more worrying than a simple exhaustion of the regeneration capacity: ecosystems may switch from sinks to net emitters of carbon.

From the point of view of the economic analysis, recognizing that pollution may be irreversible challenges the assumption, often used in optimal control and growth models (see Keeler et al. 1971, Van der Ploeg and Withagen 1991, Smulders and Gradus 1993 among others), that Nature is able to assimilate pollutants at a constant rate. Some 
authors (including Forster 1975, Comolli 1977 and Dasgupta 1982) have proposed a new formulation of the assimilation function incorporating the idea that high pollution levels drastically alter the waste assimilation capacity of Nature. Therefore, the problem becomes to assess the implications of a more sophisticated assimilation process on the optimal control of pollution (see, for instance, Forster 1975, Cesar and de Zeeuw 1994 and Tahvonen and Withagen 1996).

Tahvonen and Withagen (1996) develop an optimal control model where consumption causes a pollution stock to accumulate. They consider a decay function that is strictly concave in the reversible region and that becomes null in the irreversible region. The important point lies in the existence of a critical threshold of pollution above which the assimilation capacity becomes permanently exhausted, thereby implying an irreversible concentration of pollution. Their main result is the existence of multiple equilibria, some of them being associated with irreversible pollution. In other words, under certain circumstances, that notably involve initial conditions, it may be optimal to exhaust the environment in an irreversible manner. In the same vein, Prieur (2009) introduces the irreversibility of pollution in a general equilibrium model of growth, which gives new insights into the relationship between growth and the environment. He shows notably that the economy may be caught in highly polluted and low growth equilibrium, even when pollution abatement operates.

A noticeable shortcoming of this literature is that attention is only paid to deterministic frameworks, which means that the critical value from which the natural regeneration capacity vanishes is known to those who undertake decisions. This is of course very demanding. It seems clear that our understanding of the complexity of ecosystems is growing rapidly. But, a lot of uncertainty still surrounds natural processes and notably those that involve irreversibility. Again, if one refers to global warming, then it is highly likely that ecosystems ability to absorb carbon dioxide will disappear but the exact GHG concentration that will initiate the process is unknown. Thus, a better approach would be to consider that such a critical threshold exists but is unknown. Its precise level would be modelled as a stochastic process where the probabilities of occurrence are related to a measure of the state of the system, such as the atmospheric concentration of GHG.

Nevertheless, another stream of literature, that considers catastrophic events, deals with threshold effects related to pollution and resource management under uncertainty. Cropper (1976) takes the example of a nuclear incident whereas Tsur and Zemel (1995) focus on the potential depletion of a renewable resource. Both papers consider optimal control problems where catastrophe is a random event and the objective function is de- 
fined in terms of expectations. These expectations derive from a probability distribution function on the threshold value. In these frameworks, whenever the radioactive pollution (respectively the natural resource) stock exceeds (respectively falls below) a critical threshold, the event occurs which reduces society's utility to zero. The central question addressed by these papers is whether uncertainty about the value of the threshold may induce more conservative emission (respectively extraction) and affect consumption behaviors. It is worth mentioning that a specific and disputable assumption is made that once the threshold is reached, the system - the economy - is doomed to collapse: when the stock of nuclear waste is released, people die; when the resource is degraded, extraction and consumption cease. This particular vision largely differs from previous works on irreversible pollution that assume that whenever the pollution stock exceeds the threshold, agents cannot depend on Nature anymore to assimilate pollution but economic activity goes on. On the contrary, Tsur and Zemel (1996) and Naevdal (2006) ${ }^{1}$ consider that the event is partly reversible. There is a stock of pollution, say greenhouse gases, and Nature regenerates at constant rate. When pollution reaches the unknown threshold then the event occurs which substantially reduces the utility level. But, the economy can recover from its impact even if it implies to bear important costs (related to curing activities for instance). Tsur and Zemel (1996) notably show that when these costs are high enough then it is never optimal to maintain emission above the regeneration rate of Nature. The emission policy is more conservative than when one ignores the risk of event occurrence. Naevdal (2006) derives necessary conditions for optimal control when the threshold is a curve in n-dimensional space. It is applied to the optimal regulation of two greenhouse gases (eg. $\mathrm{CO}_{2}$ and $\mathrm{CH}_{4}$ ) that have different levels of radiative forcing and different lengths of life in the atmosphere. In particular, starting with an initial gases combination such that temperature is relatively high, it is optimal to keep temperature unchanged. Changes in emissions are then affected by the trade-off between costs and the physical properties of each gas but not by the risk associated with increases in the gases.

The question raised by the present paper is: what is the impact of uncertainty about the irreversibility threshold on optimal decisions? More precisely, since there exists a risk to overshoot the regeneration capacity of Nature, should one consume and pollute a lot provided it increases the probability of reaching the threshold, or should one adopt more conservative decisions in order to prevent the irreversible situation? In order to assess this issue, a framework is developed that encompasses the approaches in terms of catastrophic

\footnotetext{
${ }^{1}$ Naevdal and Oppenheimer (2007) extend the framework to multiple thresholds and Naevdal and Vislie (2010) focus on the role of discounting in a simplified version.
} 
events and in terms of irreversible pollution. Polluting emissions are proportional to consumption. They contribute to the degradation of the quality of the environment. Nature is able to partly recover as long as environmental quality remains above a threshold. Below this critical level, a new stage occurs where pollution becomes fully irreversible. We consider the problem of pollution control under irreversibility and uncertainty. The threshold is a random variable that is characterized by a probability distribution function such that the lower the level of environmental quality the larger the probability of hitting the threshold in the nearest future.

The analysis is based on a comparison between the non event problem - when one ignores the risk of irreversibility - and the uncertain problem - when one takes into account irreversibility but does not know the threshold value.

Within our framework, the solution to the non event problem is a balanced growth path (BGP) for consumption and environmental quality. In the case where agents are patient and/or care a lot about the environment and/or Nature regenerates at high rate, the constant growth rate is positive along the BGP and the threshold can never be met. In the opposite case, one can expect that the risk of irreversibility plays a role in the uncertain problem. Indeed, studying this problem, we show that, for a wide range of distribution functions, there exists a unique steady state, which is a saddle point. As far as the optimal policy is concerned, it implies that, for any initial level of quality above the threshold, the economy should optimally settle on the stable branch and converge toward the steady state. Thus, provided that the unknown threshold is below the long run value of environmental quality, the optimal policy is more conservative than the decisions taken when one ignores the risk of irreversibility.

Our contribution to the above mentioned literature is twofold. The analysis first reveals that economies with different fundamentals - time preference, environmental concerns may follow very distinct development paths with, or without, sustained growth in the long run. And, this result does not primarily depend on the initial level of environmental quality. Second, our conclusion is more mitigated than the ones of the literature on catastrophic events. For instance, Tsur and Zemel (1995, 1996, 2004), and Naevdal and Vislie (2010) show that, when the event is a catastrophe or is partly reversible but at huge cost, uncertainty about the threshold induces more conservative decisions because the expected loss due to the event occurrence is so high that it is never optimal to reach the threshold value. We obtain the same feature that the risk of irreversibility induces a more cautious interaction with the environment and, in general, it is sufficient to avoid irreversibility. But, in contrast with their result, we cannot rule out situations where the 
economy will optimally follow an irreversible path and consequently, will be left, in the long run, with an irreversibly degraded environment.

The paper is organized as follows: Section 2 presents the model; Section 3 provides a detailed analysis of the optimal solution when uncertainty about the irreversibility threshold is taken into account; and Section 4 concludes.

\section{The model}

We consider a partial equilibrium model where consumption, $c$, is the source of polluting emissions. Households derive utility from consumption and environmental quality, $Q$. Environmental quality can be interpreted as the quality of air or water. It can be seen as an index of biodiversity and can also be related to the atmospheric concentration of GHG. The key point being that environmental quality provides services to the economy. To capture these features, a homothetic utility function is defined over consumption and the quality of the environment:

$$
U(c, Q)=\left(c Q^{\phi}\right)^{1-\frac{1}{\sigma}} \text { with } \sigma>1, \phi>0 .
$$

This function takes value in $\mathbb{R}^{+}$, is increasing and concave with respect to $c$ and $Q$. In order for the utility to be concave in $(c, Q)$, we further assume $\varepsilon \in(0,1]$, where $\varepsilon=$ $(1+\phi)\left(1-\frac{1}{\sigma}\right)$ is the degree of homogeneity of the utility function. This, in turn, imposes a restriction on the domain of variation of the effective coefficient of relative risk aversion $(1-$ $\varepsilon) \in[0,1)$. The weight of environmental quality in preferences is given by $\frac{Q U_{Q}}{c U_{c}}=\phi>0 .{ }^{2}$ The cross derivative $U_{c Q}$ is positive which means that utility exhibits a "complementarity

\footnotetext{
${ }^{2}$ The curvature parameter $\phi$ reflects more than just ordinal preferences between $c$ and $Q$ at a point in time and since there are two arguments in the utility function, it is not immediately obvious what risk aversion means (see Debreu (1976) and Kihlstrom and Mirman (1971) for the literature on multivariate risk aversion). Equation (1) can be rewritten as:

$$
U(c, Q)=\left(c^{\frac{1}{1+\phi}} Q^{\frac{\phi}{1+\phi}}\right)^{\varepsilon} \text { with } \sigma>1, \phi>0, \varepsilon \in(0,1]
$$

Debreu (1976) calls the function in the braces the "least concave utility function". The exponents of this function may be interpreted as governing ordinal preferences between the two goods in the absence of risk. The transforming function $[\cdot]^{\varepsilon}$ can then be interpreted as governing aversion to risk. A simple calculation then reveals that the appropriate measure of risk relative aversion is $(1-\varepsilon) \geq 0$ since $\varepsilon \in(0,1]$. Following the terminology in Smith (1999) or Pommeret and Schubert (2009) we will call $(1-\varepsilon)$ the effective coefficient of relative risk aversion. Since $(1-\varepsilon)$ depends on $\phi$, environmental quality changes risk aversion.
} 
effect", in the terminology of Michel and Rotillon (1995): an increase in environmental quality increases the marginal utility of consumption and implies that households have a higher desire to consume.

Consumption causes the stock of environmental quality to deteriorate. To account for irreversibility, the dynamics of environmental quality are defined piecewise:

$$
\dot{Q}= \begin{cases}\gamma Q-c & \text { if } Q>\bar{Q} \\ -c & \text { else }\end{cases}
$$

The natural regeneration rate $\gamma \in(0,1)$ is constant and positive as long as the accumulated degradation is not too strong that is, as long as the quality remains above the irreversibility threshold $\bar{Q}$. Once the threshold is reached, a new stage occurs where the regeneration capacity is completely and permanently overwhelmed. Thus, damages become irreversible.

Note that the domain where $Q>\bar{Q}$ is called hereafter the reversible domain whereas whenever environmental quality is below or equal to $\bar{Q}$, the economy lies in the irreversible domain.

Our contribution to the literature on irreversible environmental damages is to consider that this threshold is unknown from the point of view of the policy-maker. Actually, $\bar{Q}$ is a random variable. The information regarding the location of $\bar{Q}$ is described by a probability distribution function $F(Q)=\operatorname{Pr}(\bar{Q}<Q)$ and the associated density $f(Q)=\frac{d F(Q)}{d Q}$. We do not impose any restriction on $F(Q)$ except that the shape of the distribution must convey the idea that reaching the threshold is all the more likely when environmental quality is low. Let us define the hazard rate $\Lambda(Q)$ as follows:

$$
\Lambda(Q)=\frac{f(Q)}{F(Q)},
$$

this requires the hazard rate to be monotone non increasing: $\Lambda^{\prime}(Q) \leq 0$.

The probability to reach the threshold at some date $t+d t$, provided that $Q(t)>\bar{Q}$, is monotically decreasing in $Q$. Again, it seems quite reasonable to consider that the probability to hit the threshold provided it has not been reached yet is decreasing with the level of environmental quality.

\section{The emission problem}

The present work investigates the impact of uncertainty about the value of the irreversibility threshold on the optimal emission policy. For that purpose, the analysis is based on a 
comparison between the non event problem - when one ignores the risk of irreversibility and the uncertain problem - when one takes into account irreversibility but does not know the threshold value. We notably wonder if taking into account uncertainty affects the solution by inducing more conservative decisions regarding emissions. Clearly, uncertainty would not bring much to the analysis in the situation where the system is "well-behaved" that is, where the optimal solution is accompanied by nondecreasing trajectories for environmental quality (see for e.g. Ayong Le Kama and Schubert 2004).

This raises the important question of when uncertainty and irreversibility play a role. Answering this question requires looking carefully at the non event problem.

\subsection{The non event problem}

When the policy maker does not take into account the risk of irreversibility, the optimal policy is a sequence $\{c(t)\}_{t=0}^{\infty}$ that solves:

$$
\begin{aligned}
& \max _{\{c\}} \int_{0}^{+\infty} \exp ^{-\rho t} U(c, Q) d t \\
& \text { s.t } \dot{Q}=\gamma Q-c ; Q(0)=Q_{0}
\end{aligned}
$$

with $\rho \in(0,1)$, the discount rate.

From the resolution, one can easily check that both consumption and environmental quality follow a balanced growth path with positive growth if and only if $\rho-\gamma(1+\phi) \leq 0$ (see Ayong Le Kama 2001 and Ayong Le Kama and Schubert 2004). For nondecreasing trajectories, the issue of irreversibility is never raised. It means that it is known with certainty that the threshold will never be exceeded.

Therefore, the most interesting case is the one where the solution to the non event problem generates nonincreasing trajectories for the quality, which may then fall below the irreversible threshold. In that case solely, we shall be able to provide new insights into the role of uncertainty about $\bar{Q}$. Considering this case requires the condition $\rho-\gamma(1+\phi)>0$ to be imposed. This condition encompasses situations where $i /$ the discount rate is sufficiently high and $i$ / the assimilation capacity of Nature and/or environmental concern is relatively low. It has the following consequences on decisions and the environment. Starting with a level of environmental quality above the threshold, assume that agents are impatient and that the value attached to the environment is lower than the one placed on consumption. It implies that priority is given to consumption at the expense of future environmental quality. High levels of consumption are in turn associated with high emissions. If, in addition, Nature regenerates itself at low rate, then emissions exceed the amount of pollution assimilated by Nature at each time, which means that environmental 
quality decreases. Because of the complementarity effect, the fall in quality finally causes consumption to decrease too.

\subsection{The emission problem under uncertainty and irreversibility}

In the remainder of the paper, attention is paid to the least favourable conditions for environmental conservation $(\rho-\gamma(1+\phi)>0)$. Under these conditions, we assess the properties of the optimal policy under both uncertainty and irreversibility. To deal with this issue, a two-step analysis is conducted that mainly consists in solving the problem recursively. The intertemporal optimization program is decomposed into two successive subprograms, depending on whether the threshold has already been reached. First, we consider that the economy starts, at some date $\tau$, with a level of environmental quality equal to $\bar{Q}$ and assess the subproblem of maximizing discounted utility from $t=\tau$ onwards. Then, the overall problem is to determine optimal consumption paths given any $Q_{0}>\bar{Q}$. It requires to incorporate, in the objective function, the value function associated with the irreversible problem when $Q(\tau)=\bar{Q}$. Due to the specification used for the utility function, the focus is put on interior solution that is, the control variable $c$ is assumed to be positive.

\subsubsection{The post event problem}

Assume first that the threshold has been reached at date $t=\tau$. In this case, there is no uncertainty anymore since the policy maker knows that the situation has turned irreversible. The optimization program can be written as:

$$
W\left(Q_{\tau}\right)=\left\{\begin{array}{l}
\max _{\{c\}} \int_{\tau}^{\infty} \exp ^{-\rho(t-\tau)} U(c, Q) d t \\
\text { s.t. } \dot{Q}=-c ; Q_{\tau}=\bar{Q}
\end{array}\right.
$$

The Hamiltonian in current value, with $\pi \geq 0$ the co-state variable associated with $Q$ :

$$
H(c, Q, \pi)=U(c, Q)-\pi c
$$

Necessary conditions read, for interior solutions:

$$
\begin{aligned}
U_{c}= & \leftrightarrow\left(1-\frac{1}{\sigma}\right) \frac{\left(c Q^{\phi}\right)^{1-\frac{1}{\sigma}}}{c}=\pi \\
\dot{\pi} & =\rho \pi-U_{P} \\
\leftrightarrow & \dot{\pi}=\rho \pi-\phi\left(1-\frac{1}{\sigma}\right) \frac{\left(c Q^{\phi}\right)^{1-\frac{1}{\sigma}}}{Q}
\end{aligned}
$$


and the transversality condition:

$$
\lim _{t \rightarrow \infty} \exp ^{-\rho t} \pi(t) Q(t)=0
$$

The first optimality condition (4) states that the marginal utility of consumption is equal to the shadow price of environmental quality. This also means that the benefits from an additional unit of consumption (LHS) should be equal to the future decrease in utility which that consumption entails (RHS). The second condition is the equation of motion of the co-state variable $\pi$.

Differentiating eq. (4) and using (5), we obtain the following system of differential equations:

$$
\left\{\begin{array}{l}
\frac{\dot{c}}{c}=-\sigma\left[\rho-\frac{\phi}{\sigma} \frac{c}{Q}\right] \\
\dot{Q}=-\frac{c}{Q}
\end{array}\right.
$$

that characterizes the dynamics in the irreversible region.

Proposition 1 In the irreversible domain, the economy follows a BGP where consumption and environmental quality grow at rate $g^{I}=-\frac{\sigma \rho}{1+\phi}<0$. Along the BGP, the pair $\left(C_{\tau}(t), Q_{\tau}(t)\right)$ is given by:

$$
Q_{\tau}(t)=Q_{\tau} \exp ^{-\frac{\sigma \rho}{1+\phi}(t-\tau)} \text { and } C_{\tau}(t)=\frac{\sigma \rho}{1+\phi} Q_{\tau} \exp ^{-\frac{\sigma \rho}{1+\phi}(t-\tau)}
$$

Proof. See the appendix A.

It means that once the economy has reached the irreversible domain, it is optimal to fully deteriorate the stock of environmental quality. The process is accompanied in turn by a perpetual reduction of the consumption level.

We retrieve the maximum value associated with the optimal policy (see the appendix A),

$$
W\left(Q_{\tau}\right)=\frac{1}{\sigma \rho}\left(\frac{\sigma \rho}{1+\phi} Q_{\tau}^{1+\phi}\right)^{1-\frac{1}{\sigma}}>0 \text { for any possible } Q_{\tau},
$$

since it will be used as a scrap value function in the second step of the analysis, where the intertemporal emission problem "before" irreversibility is studied.

\subsubsection{The uncertain problem}

The same emission problem is examined assuming now that the economy starts with a level of quality strictly above the threshold. In the reversible domain, uncertainty clearly matters, which means that the valuation function, for planning, is defined in terms of 
expectations. More precisely, given that the "event" has not occurred at time $t=0$, the intertemporal consumption/emission problem entails finding a policy $\{c(t)\}_{t=0}^{\infty}$ that solves:

$$
\begin{aligned}
& \max E U=E_{\tau}\left\{\int_{0}^{\infty} \exp ^{-\rho t} U(c, Q) d t \text { । } \tau>0\right\} \\
& \text { s.t } \dot{Q}=\gamma Q-c ; Q_{0}>\bar{Q} \text { given }
\end{aligned}
$$

where $E_{\tau}$ corresponds to expectations with respect to the distribution of $\tau,\left\{f_{\tau}(t), F_{\tau}(t)\right\}$, the uncertain time when the threshold is reached.

Let us first have a look to the expression of the expected utility. For any distribution of $\tau$, following Dasgupta and Heal (1974), by integrating the valuation function in (8), yields:

$$
E U=\int_{0}^{\infty} \exp ^{-\rho t}\left[U(c, Q)\left(1-F_{\tau}(t)\right)+f_{\tau}(t) W\left(Q_{\tau}\right)\right] d t
$$

with $1-F_{\tau}(t)=\operatorname{Pr}(\tau>t)$, the probability to remain reversible until date $t$ and $f_{\tau}(t)$, the probability to turn irreversible around $t$.

The expected utility thus can be expressed as the combination of two terms. The first term is the level of utility obtained from consuming and enjoying quality as long as quality remains reversible that is, with a probability $1-F_{\tau}(t)$. The second term is the preceding value $W\left(Q_{\tau}\right)$ of the emission problem starting at the date when damages become irreversible. It is weighted by the probability to reach the threshold at some date close to $t$.

Now, it is worth mentioning that the distribution of $\tau$ is not any distribution. Actually, $\tau$ is a random variable whose distribution is derived from the distribution of $\bar{Q}$ (see Naevdal, 2010). And it is not immediately obvious what this distribution is. This is mainly because the history matters, which means that as the process evolves in time, so does the irreversibility probability. At any time $t$, the distribution of $\bar{Q}$ depends on the whole trajectory of $Q$ up to $t$ and, in particular, on $\hat{Q}(t)=\min _{\tau \in[0, t)} Q(\tau)$. This feature has an important implication on the definition of expectations. Given that irreversibility has not occurred at some time before $t$, the hazard rate is nil: $\Lambda(Q(t))=0$ for any $Q(t) \geq \hat{Q}(t)$. In other words, irreversibility may only occur for $Q(t)<\hat{Q}(t)$. This substantially complicates the way expectations have to be defined.

However, the problem becomes simpler when one works with monotone trajectories $\{Q(t)\}_{t=0}^{\infty}$, for the state variable. That is why we have chosen to pay attention only to monotone trajectories of the state variable $Q(t)$. Our approach relies on Tsur and Zemel (1995) who establish, in a problem similar to ours, that at least one of the optimal state trajectories corresponding to problem (8) evolves monotonically in time (see their proposition 2.1). 
What kind of monotone trajectories should we deal with? Assume that some trajectory is monotone nondecreasing. It means that the policy maker knows that the threshold will never be hit. It turns, it generates a zero hazard rate. In other words, one has to replace $f_{\tau}(t)=0$ and $1-F_{\tau}(t)=1$ in $(9)$ and the valuation function becomes similar to the one of the non event problem. But, in this case, we have shown (see proposition 1) that the optimal policy is accompanied by a decreasing trajectory for $Q$. Thus, there is a contradiction. It means that monotone trajectories associated with our problem are necessarily nonincreasing.

For nonincreasing trajectories of $Q$, it is easy to characterize the distribution of $\tau$ :

$$
1-F_{\tau}(t)=\operatorname{Pr}(\tau>t \text { । } \tau>0)=\operatorname{Pr}\left(Q(t)>\bar{Q} \text { । } Q_{0}>\bar{Q}\right)=\frac{F(Q(t))}{F\left(Q_{0}\right)},
$$

and the density function:

$$
f_{\tau}(t)=-\frac{\dot{Q} f(Q)}{F\left(Q_{0}\right)}=\frac{(c-\gamma Q) f(Q)}{F\left(Q_{0}\right)} .
$$

It is worth noting that the hazard rate $\Lambda_{\tau}(t)=\Lambda(Q)(c-\gamma Q)$ depends not only on $Q$ but also on its rate of change, $\dot{Q}$.

By substituting equations (10) and (11) into (9), the optimization program can be rewritten as: ${ }^{3}$

$$
W\left(Q_{0}\right)=\left\{\begin{array}{l}
\max _{\{c\}} \frac{1}{F\left(Q_{0}\right)} \int_{0}^{\infty} \exp ^{-\rho t}[U(c, Q) F(Q)+f(Q)(c-\gamma Q) W(Q)] d t \\
\text { s.t } \dot{Q}=\gamma Q-c ; Q_{0}>\bar{Q} \text { given }
\end{array}\right.
$$

\subsubsection{Equilibrium analysis}

Once rewritten, the problem is a standard optimal control problem that can be solved by using "standard" techniques. In addition, it belongs to the specific class of infinitehorizon and autonomous problems that produce optimal trajectories, for the state variable $Q$, which are indeed monotone.

The Hamiltonian in current value can be expressed as follows:

$$
H(c, Q, \lambda)=U(c, Q) F(Q)+f(Q)(c-\gamma Q) W(Q)+\lambda(\gamma Q-c),
$$

\footnotetext{
${ }^{3}$ Since attention is paid to nonincreasing trajectories, this program has an implicit additional restriction which is: $\dot{Q} \leq 0$. Taking explicitly into account this non-negativity constraint would require working with a Lagrangian rather than a Hamiltonian and studying the corner solution with $\dot{Q}=0$. However, since this case does not bring much to our analysis, it has been left aside. Details about this case are available upon request.
} 
with $\lambda$ the co-state variable associated with $Q$.

Necessary conditions are given by:

$$
\begin{gathered}
U_{c} F(Q)+W(Q) f(Q)=\lambda \\
\dot{\lambda}=(\rho-\gamma) \lambda-\left(U_{Q} F(Q)+U f(Q)\right)-\left(f^{\prime}(Q)(c-\gamma Q)-\gamma f(Q)\right) W(Q)-f(Q)(c-\gamma Q) W^{\prime}(Q),
\end{gathered}
$$

and the transversality condition:

$$
\lim _{t \rightarrow \infty} \exp ^{-\rho t} \lambda(t) Q(t)=0
$$

According to (12), the marginal utility of consumption is weighted by the probability to lie in the reversible domain. Compared with (4), the LHS of the first optimality condition is augmented with a second term that reflects that an additional unit of consumption also changes the probability to switch regime and earn the value $W(Q)$. The equation of motion of the co-state variable (13) is also modified and notably accounts for the fact that changes in environmental quality have an impact on the expected utility the agent gets from consuming and enjoying quality. The trade-off faced by the policy maker is pretty simple. Maintaining high levels of consumption and emissions enhances immediate benefits. But, at the same time, it increases the probability to reach the irreversibility threshold and to switch regime.

Differentiating eq. (12), and using eq. (13) yield:

$$
\frac{\dot{c}}{c}=\sigma\left[\begin{array}{l}
\left(\Lambda(Q) Q+\phi\left(1-\frac{1}{\sigma}\right)\right)\left(\gamma+\frac{c}{Q} \frac{1}{\sigma}\left(1-\frac{1}{\sigma}\right)^{-1}\right)- \\
-(\rho-\gamma)-\frac{1}{\sigma}\left(1-\frac{1}{\sigma}\right)^{-1} \Lambda(Q)\left(\frac{\sigma \rho Q}{1+\phi}\right)^{1-\frac{1}{\sigma}} c^{\frac{1}{\sigma}}
\end{array}\right]
$$

which, together with the law of motion of the environmental quality

$$
\dot{Q}=\gamma Q-c,
$$

characterize the system of differential equations.

The first consequence of uncertainty is that constant and perpetual growth no longer is possible. Thus, we emphasize the existence of steady states as long run equilibrium outcomes.

In a steady state, from (15) and $Q=0$, we have $c=\gamma Q$. Substituting this relation into (14), a steady state is a value $Q$ that solves:

$$
Q \Lambda(Q)\left(1-\left(\frac{\rho}{\gamma(1+\phi)}\right)^{1-\frac{1}{\sigma}}\left(\frac{1}{\sigma}\right)^{\frac{1}{\sigma}}\right)=(1+\phi)\left(1-\frac{1}{\sigma}\right)\left(\frac{\rho}{\gamma(1+\phi)}-1\right)
$$


Note that we consider a wide range of distribution functions, which includes Weibull, Rayleigh, exponential and log-logistic distributions. For instance,

$$
\begin{aligned}
& \text { Weibull, for } k \geq 1, \delta>0: \Lambda(Q)=\frac{\frac{k}{\delta}\left(\frac{Q}{\delta}\right)^{k-1} \exp ^{-\left(\frac{Q}{\delta}\right)^{k}}}{1-\exp ^{-\left(\frac{Q}{\delta}\right)^{k}}} \\
& \text { Log-logistic, } \alpha>0, \beta \geq 1: \Lambda(Q)=\frac{\beta}{\alpha} \frac{\left(\frac{Q}{\alpha}\right)^{-\beta-1}}{\left(1+\left(\frac{Q}{\alpha}\right)^{-\beta}\right)}
\end{aligned}
$$

For this class of distributions, the existence result is established in proposition 2.

Proposition 2 The necessary condition for the existence of a unique steady state, denoted by $\left(c^{*}, Q^{*}\right)$, is:

$$
\rho<\gamma(1+\phi) \sigma^{\frac{1}{\sigma-1}}
$$

A sufficient existence condition is

$$
\rho<\gamma(1+\phi) \hat{\chi}
$$

with $\hat{\chi}$ solution of:

$$
1-\frac{1}{\sigma} \chi^{1-\frac{1}{\sigma}}-(1+\phi)\left(1-\frac{1}{\sigma}\right) \chi+(1+\phi)\left(1-\frac{1}{\sigma}\right)=0
$$

Proof. See the appendix B.

The inequality (16) involves all the fundamentals of the economy. Recall that we have considered the least favourable conditions for environmental conservation: $\rho-\gamma(1+$ $\phi)>0$. This additional condition states that the regeneration capacity of Nature and/or environmental concern should not be too low vis-?-vis the discount rate. In other words, the agents should care sufficiently about the environment and about the future in order for the economy to be able to reach a steady state in the long run. Compared with the initial restriction, $\rho-\gamma(1+\phi)>0$, one may note that the elasticity of substitution parameter $\sigma$ appears. This opens the door to an alternative interpretation in terms of risk aversion. Indeed, the condition (16) is all the most likely to hold if $\sigma$ is close to one, which is equivalent to requiring that the effective coefficient of relative risk aversion $(1-\varepsilon) \in[0,1)$ is close to its upper bound. It means that the existence of the steady state requires risk aversion to be high enough. Note that the necessary condition (16) is also sufficient provided that the shape parameter of the distribution ( $k$, for the Weibull distribution, $\beta$, for the log-logistic) is chosen appropriately. Otherwise, one can impose the technical condition (17), which is sufficient for existence.

As for the stability properties, results are summarized in the following proposition.

Proposition 3 The steady state $\left(c^{*}, Q^{*}\right)$ is a saddle point. 
Proof. See the appendix C.

The saddle point stability implies that there exists a stable manifold. For any $Q_{0}>\bar{Q}$, it is thus possible to pick up an initial level of consumption that places the economy on the stable branch, which means that it will converge, in the long run, toward the high steady state. There also exists an unstable manifold along which the economy diverges from the equilibrium. Under regular conditions, the optimal policy then consists in appropriately choosing initial consumption so that $(c(0), Q(0))$ is located on the stable branch of the saddle point.

At first, these results may seem quite optimistic. They emphasize that depending on whether the policy maker takes into account irreversibility, development paths exhibit very distinct features. Indeed, when one ignores irreversibility, the economy follows a path that leads to the complete exhaustion of the environment (see proposition 1). It seems that this is no longer the case when the policy maker cares about the risk since the long run equilibrium is a steady state with positive quality. But, things may not be so simple when there is a risk of irreversibility and the location of the steady state now matters.

Investigating more deeply the kind of optimal trajectories the uncertain problem may exhibit is the purpose of the next section.

\section{Reversible versus irreversible optimal policy}

In this section, we wonder if the decisions taken by the policy maker necessarily protect the economy against the irreversible degradation of the environment. Actually, the important question raised by our analysis is whether the optimal policy is "reversible". It turns out that the location of the steady state with respect to the unknown threshold is crucial for the discussion about the properties of the optimal policy. Two opposite cases are depicted in Fig. 1 and 2.

Suppose first that the irreversibility threshold is low enough: $Q^{*}>\bar{Q}$. This situation is depicted in Fig.1. In this case, the economy will reach in the long run the steady state by following path (1). ${ }^{4}$ This path corresponds to the optimal reversible policy since environmental quality always remains above the threshold during the convergence toward the saddle point. Starting with an initial level of environmental quality that is high enough, $Q_{0}>Q^{*}$, the planner will optimally let consumption and environmental quality decrease along path (1) until they reach their long run values. When $\rho>\gamma(1+\phi)$, the optimal

\footnotetext{
${ }^{4}$ Note that path $(2)$, when $Q_{0}<Q^{*}$, is irrelevant for our analysis since attention has only been paid to nonincreasing trajectories for environmental quality (see also footnote 2 ).
} 
policy of the non event problem consists of a continuous depletion of environmental quality. When one accounts for uncertainty and irreversibility, the policy still is accompanied by a decrease in environmental quality - at each date, consumption is above the regeneration rate of Nature - but the planner seeks to reduce the risk of facing an irreversibly degraded environment, which is actually sufficient to avoid irreversibility. Thus, taking into account the risk of irreversibility induces more conservative decisions in terms of consumption and polluting emissions. This is the most optimistic scenario that shares similarities with conclusions of the literature on catastrophic events (see Tsur and Zemel, 1995).

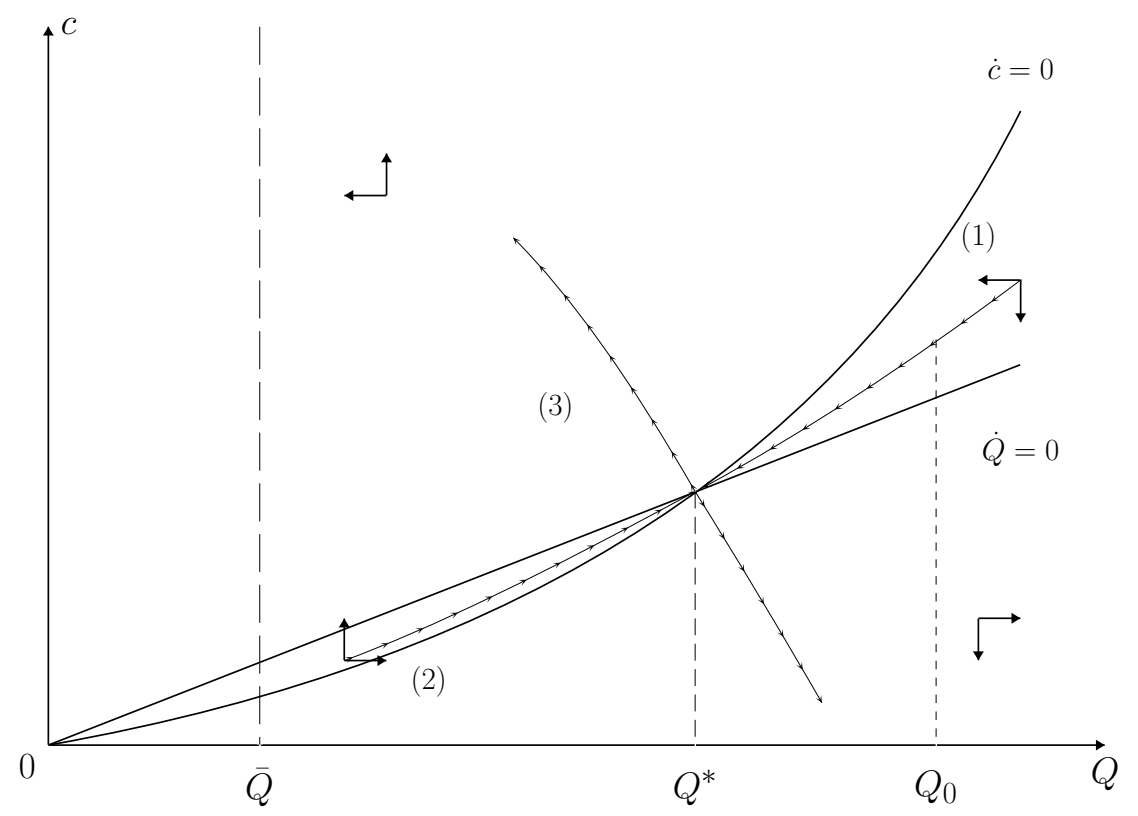

Figure 1: Convergence patterns: optimal reversible policy

However, there is another scenario in which irreversible degradation of this environment is unavoidable. This situation occurs when the unknown threshold is higher than the equilibrium value of quality: $Q^{*}<\bar{Q}$ and lies between the initial condition and the long run value of environmental quality. Fig.2 illustrates what happens in this case. The planner chooses initial consumption so that the economy follows path (1), which normally leads the system to the steady state. But, along path (1), the economy will necessarily reach the threshold $\bar{Q}$ in finite time. It means that the optimal policy is to pursue the solution of problem (8) only until the irreversibility threshold is hit. At this date, there is a switch in 


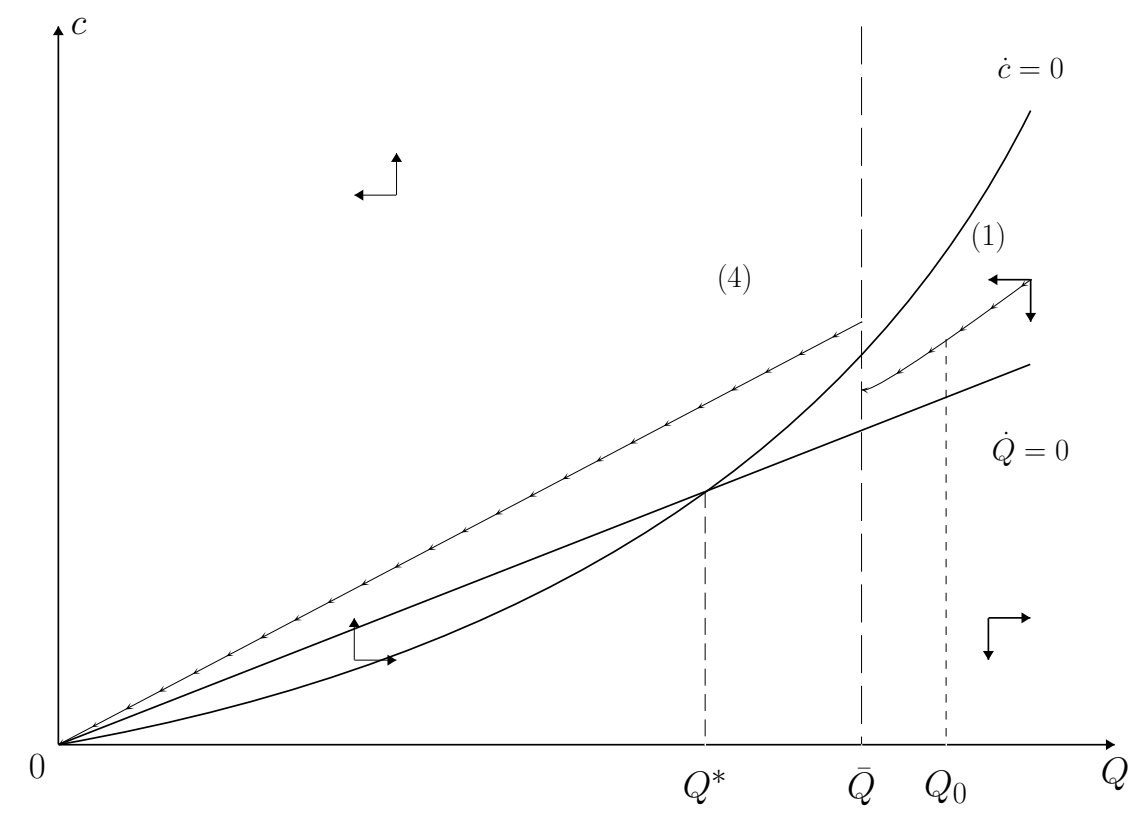

Figure 2: Convergence patterns: optimal irreversible policy.

regime and the optimal policy from then on is to follow path (4), which is the solution of the post event problem (see proposition 1). The economy settles on the BGP accompanied by a perpetual decrease of environmental quality and consumption, both asymptotically converging toward zero. Note that there is a jump in consumption when environmental damages become irreversible. In this case, preventing the risk of irreversibility admittedly forces the economy to adopt a more cautious behavior in interacting with the environment. But, at the same time, the incentive is to maintain a high level of consumption, which goes with the degradation of environmental quality. Finally, the former effect is not enough to compensate for the latter particularly as irreversibility occurs for a level of quality that is relatively high. This result contrasts with Tsur and Zemel (1996)'s conclusion that when the event (substantial decrease in utility) is partly reversible, important curing costs (borne to recover from the event's impact) are sufficient to avoid the risk of catastrophe.

Remark. In the particular case where there is no steady state (condition (16) does not hold), starting from a state located above the $\dot{Q}=0$ locus, the economy will necessarily reach the irreversible domain. It means that the only possible solutions correspond to irreversible paths. 


\section{Conclusion}

We consider an optimal consumption and pollution problem that has two important features. Environmental damages due to economic activities may be irreversible and the level at which the degradation becomes irreversible is unknown. Particular attention is paid to the situation where agents are relatively impatient and/or do not care a lot about the environment and/or Nature regenerates at low rate. We show that the optimal policy of the uncertain problem drives the economy in the long run toward a steady state while ignoring irreversibility would doom the economy to suffer from an irreversibly and completely degraded environment. Therefore, accounting for the risk of irreversibility induces more conservative decisions regarding consumption and polluting emissions. So, our work contributes to the literature on catastrophic events surveyed by Tsur and Zemel (2004) by extending its main result to a new risk related to ecological processes. Indeed, Tsur and Zemel (1995), in their resource management problem, show that the expected loss due to the event occurrence is so high that it is never optimal to reach the threshold value. Tsur and Zemel (1996) get the same conclusion when they adopt a less extreme approach that consists in considering that the economy does not collapse once the threshold is reached but that recovering from the catastrophe is very costly. In contrast with this conclusion, in general, we cannot rule out situations where the economy will optimally follow an irreversible path and consequently, will be left, in the long run, with an irreversibly degraded environment. In that sense, our result also echoes the conclusions of Tahvonen and Withagen (1996), obtained in the case where the irreversibility threshold is known with certainty.

Our results provide strong support for improving knowledge about ecological processes. Indeed, because of the lack of information, the policy maker may adopt decisions that drive the economy to an irreversible world in the long run. In the particular case of interest, scientific progress may help solving the uncertainty surrounding the value of the threshold. Therefore, a natural extension of this work would consist in introducing a kind of learning process where the arrival of new information would change the distribution of the threshold in such a way that the policy maker would obtain a finer and finer description of the value at which irreversibility occurs. 


\section{References}

[1] Ayong Le Kama, A.: Preservation and exogenous uncertain future preferences. Economic Theory 18, 745-752 (2001).

[2] Ayong Le Kama, A. and Schubert, K.: Growth, environment and uncertain future preferences. Environmental and Resource Economics 28, 31-53 (2004).

[3] Cesar, H., de Zeeuw, A.: Substainability and the greenhouse effect: Robustness analysis of the assimilation function. In Control and Game Theoretical Models of the Environment, J. Filar and C. Carraro Eds, Birkh?user, Boston (1994)

[4] Comolli, P.: Pollution control in a simplified general equilibrium model with production externalities. Journal of environmental Economics and Management 4, 289-304 (1977)

[5] Cropper, M.L: Regulating activities with catastrophic environmental effects. Journal of environmental Economics and Management 3, 1-15 (1976)

[6] Dasgupta, P.: The Control of Resources. Basil Blackwell, Oxford (1982)

[7] Dasgupta, P., Heal, G: The optimal depletion of exhaustible resource. The Review of Economic Studies 41, 3-28 (1974)

[8] Dasgupta, P., M?ler, K-G.: The economics of non-convex ecosystems: introduction. Environmental and Resource Economics 26, 499-525 (2003)

[9] Debreu, G.: Least concave utility functions, Journal of Mathematical Economics 8, 353-394 (1976).

[10] Forster, B.: Optimal pollution control with nonconstant exponential rate of decay. Journal of environmental Economics and Management 2, 1-6 (1975)

[11] Intergovernmental Panel on Climate Change, Technical summary: "Climate change 2007: Impact, adaptation and vulnerability. Contribution of working group II to the fourth assessment report of the IPCC (2007)

[12] Keeler, E., Spence, M., Zeckauser, R.: The optimal control of pollution. Journal of Economic Theory 4, 19-34 (1971)

[13] Khilstrom, R., Mirman L.: Risk aversion with many commodities. Journal of Economic Theory 8, 361-368 (1974). 
[14] Michel, P. and Rotillon, G.: Desutility of pollution and endogenous growth. Environmental and Resource Economics 6, 279-300 (1995)

[15] Naevdal, E. : Dynamic optimization in the presence of threshold effects when the location of the threshold is uncertain -with an application to a possible disintegration of the Western Antartic Ice Sheet. Journal of Economic Dynamics and Control 30, 1131-1158 (2006).

[16] Naevdal, E. and Oppenheimer, M. : The economics of the thermohaline circulation -A problem with multiple thresholds of unknown locations. Resource and Energy Economics 29, 262-283 (2007).

[17] Naevdal, E. and Vislie, J. : Climate change, catastrophic risk and the relative unimportance of discounting. mimeo (2010).

[18] Pommeret, A. and Schubert, K.: Abatement technology adoption under uncertainty. Macroeconomic Dynamics 13(4), 493-522 (2009)

[19] Prieur, F.: The environmental Kuznets curve in a world of irreversibility. Economic Theory 40(1), 57-90 (2009).

[20] Smith, W.T.: Risk, the spirit of capitalism and growth: the implications of a preference for capital. Journal of Macroeconomics 21, 241-262 (1999).

[21] Smulders, S., Gradus, R.: The Trade-off between Environmental Care and Longterm Growth: Pollution in three Prototype Growth Models. Journal of Economics 58, 25-51 (1993)

[22] Tahvonen, O., Withagen, C.: Optimality of irreversible pollution accumulation. Journal of Economic Dynamics and Control 20, 1775-1795 (1996)

[23] Tsur, Y., Zemel, A.: Uncertainty and irreversibility in groundwater resource management. Journal of environmental Economics and Management 29, 149-161 (1995)

[24] Tsur, Y., Zemel, A.: Accounting for global warming risks: Resource management under event uncertainty. Journal of Economic Dynamics and Control 20, 1289-1305 (1996)

[25] Tsur, Y., Zemel, A.: Endangered aquifers: Groundwater management under threats of catastrophic events. Water Resources Research 40, 1-10 (2004) 
[26] Van der Ploeg, F., Withagen, C.: Pollution Control and the Ramsey Problem. Environmental and Resources Economics 1, 215-236 (1991) 


\section{Appendix}

\section{A Existence of a balanced growth path (proof of prop.1)}

Within the irreversible domain, the dynamics are described by the following system:

$$
\begin{gathered}
\frac{\dot{c}}{c}=-\sigma\left[\rho-\frac{\phi}{\sigma} \frac{c}{Q}\right] \\
\frac{\dot{Q}}{Q}=-\frac{c}{Q}
\end{gathered}
$$

Because of the specific utility function we consider, conditions for the existence of a balanced growth path (BGP), where consumption and the environment grow at the same constant rate, are met. Actually, define $x$ as the ratio between $c$ and $Q: x=\frac{c}{Q}$ which implies that $\frac{\dot{x}}{x}=\frac{\dot{c}}{c}-\frac{\dot{Q}}{Q}$. Equation (18) becomes then:

$$
\frac{\dot{x}}{x}=-\sigma \rho+(1+\phi) x
$$

The stationary solution to (19) is:

$$
x^{I *}=\frac{\sigma \rho}{1+\phi}>0
$$

The economy directly settles on a balanced growth path. Since $\frac{\dot{c}}{c}=\frac{\dot{Q}}{Q}=-x^{I *}$, consumption and the environment decrease at the constant rate $x^{I *}$ and asymptotically converge to zero.

Denote by $\left(C_{\tau}(t), Q_{\tau}(t)\right)$ (resp. by $W\left(Q_{\tau}\right)$ ) the optimal solution (resp. the maximum value) in the irreversible domain. Optimality candidates are:

$$
Q_{\tau}(t)=Q_{\tau} \exp ^{-\frac{\sigma \rho}{1+\phi}(t-\tau)} \text { and } C_{\tau}(t)=\frac{\sigma \rho}{1+\phi} Q_{\tau} \exp ^{-\frac{\sigma \rho}{1+\phi}(t-\tau)}
$$

For these candidates, the term inside the integral reads:

$$
\exp ^{-\rho(t-\tau)} U\left(C^{\tau}(t), Q^{\tau}(t)\right)=\theta_{\tau} \exp ^{-\sigma \rho t} \text { with } \theta_{\tau}=\exp ^{\sigma \rho \tau}\left(\frac{\sigma \rho}{1+\phi} Q_{\tau}^{1+\phi}\right)^{1-\frac{1}{\sigma}}>0
$$

and one can easily verify that the value $W\left(Q_{\tau}\right)$ is finite, with:

$$
W\left(Q_{\tau}\right)=\theta_{\tau} \frac{\exp ^{-\sigma \rho \tau}}{\sigma \rho}=\frac{1}{\sigma \rho}\left(\frac{\sigma \rho}{1+\phi} Q_{\tau}^{1+\phi}\right)^{1-\frac{1}{\sigma}}>0 .
$$




\section{B Existence of a steady state (proof of prop. 2)}

Studying existence boils down to finding the conditions under which the following equation,

$$
Q \Lambda(Q)\left(1-\left(\frac{\rho}{\gamma(1+\phi)}\right)^{1-\frac{1}{\sigma}}\left(\frac{1}{\sigma}\right)^{\frac{1}{\sigma}}\right)=(1+\phi)\left(1-\frac{1}{\sigma}\right)\left(\frac{\rho}{\gamma(1+\phi)}-1\right)
$$

has a solution.

Apart from properties of the distribution function, it turns out that the inequality

$$
1-\left(\frac{\rho}{\gamma(1+\phi)}\right)^{1-\frac{1}{\sigma}}\left(\frac{1}{\sigma}\right)^{\frac{1}{\sigma}}>0 \leftrightarrow \rho<\gamma(1+\phi) \sigma^{\frac{1}{\sigma-1}}
$$

is a necessary condition of existence.

We consider a class of distribution functions (Weibull, Rayleigh, exponential and loglogistic distributions) that all exhibit the two following features: $\Lambda^{\prime}(Q)<0,(\Lambda(Q) Q)^{\prime}<0$ for all $Q>0$. In addition, one can easily check that, for each and every distribution:

$$
\lim _{Q \rightarrow 0} Q \Lambda(Q) \geq 1 \text { and } \lim _{Q \rightarrow \infty} Q \Lambda(Q)=0
$$

Thus, in order to guarantee existence, it is sufficient to impose

$$
\left(1-\left(\frac{\rho}{\gamma(1+\phi)}\right)^{1-\frac{1}{\sigma}}\left(\frac{1}{\sigma}\right)^{\frac{1}{\sigma}}\right)>(1+\phi)\left(1-\frac{1}{\sigma}\right)\left(\frac{\rho}{\gamma(1+\phi)}-1\right)
$$

Define $\chi$ as follows: $\chi=\frac{\rho}{\gamma(1+\phi)}$. Then, eq. (20) is equivalent to

$$
H(\chi)=1-\left(\frac{1}{\sigma}\right)^{\frac{1}{\sigma}} \chi^{1-\frac{1}{\sigma}}-(1+\phi)\left(1-\frac{1}{\sigma}\right) \chi+(1+\phi)\left(1-\frac{1}{\sigma}\right)>0 .
$$

Having a look at the properties of $H(\chi)$, one can check, remembering that $H(\chi)$ is defined on $] 1, \infty\left[\right.$ (because of our restriction $\rho>\gamma(1+\phi)$ ) that: $\lim _{\chi \rightarrow 1^{+}} H(\chi)=$ $1-\left(\frac{1}{\sigma}\right)^{\frac{1}{\sigma}}>0, \lim _{\chi \rightarrow \infty} H(\chi)=-\infty, H^{\prime}(\chi)=-\left(1-\frac{1}{\sigma}\right)\left(\frac{1}{\sigma}\right)^{\frac{1}{\sigma}} \chi^{-\frac{1}{\sigma}}-(1+\phi)\left(1-\frac{1}{\sigma}\right)<0$ and $H^{\prime \prime}(\chi)=\frac{1}{\sigma}\left(1-\frac{1}{\sigma}\right)\left(\frac{1}{\sigma}\right)^{\frac{1}{\sigma}} \chi^{-\frac{1}{\sigma}-1}>0$ for all $\chi>1$. It means that $\exists$ ! $\hat{\chi}$ such that $H(\hat{\chi})=0$ and $H(\chi)>0 \forall \chi<\hat{\chi}$.

To sum up, imposing $\chi<\hat{\chi}$ which is equivalent to $\rho<\gamma(1+\phi) \hat{\chi}$ is sufficient for existence. 


\section{Local stability of the steady state (proof of prop. 3)}

From the linearization of the system of differential equations,

$$
\left\{\begin{array}{l}
\dot{c}=G(c, Q) \\
Q=\gamma Q-c
\end{array},\right.
$$

we get the Jacobian:

$$
J=\left[\begin{array}{ll}
G_{c}\left(c^{*}, Q^{*}\right) & G_{Q}\left(c^{*}, Q^{*}\right) \\
-1 & \gamma
\end{array}\right]
$$

The two roots $\left(\mu_{1}, \mu_{2}\right)$ of the characteristic polynomial verify: $\mu_{1}+\mu_{2}=\operatorname{traJ}$ and $\mu_{1} \mu_{2}=\operatorname{det} J$.

From (14), note that $G(c, Q)$ can be rewritten as:

$$
G(c, Q)=\sigma c\left[\left(\Lambda(Q) Q+\phi\left(1-\frac{1}{\sigma}\right)\right)\left(\gamma+\frac{c}{Q} \frac{1}{\sigma}\left(1-\frac{1}{\sigma}\right)^{-1}\right)-(\rho-\gamma)-\frac{1}{\sigma}\left(1-\frac{1}{\sigma}\right)^{-1} \Lambda(Q)\left(\frac{\sigma \rho Q}{1+\phi}\right)^{1-\frac{1}{\sigma}} c^{\frac{1}{\sigma}}\right]
$$

Direct calculations yield:

$$
\begin{aligned}
& G_{c}\left(c^{*}, Q^{*}\right)=\rho-\gamma \\
& G_{Q}\left(c^{*}, Q^{*}\right)=\gamma\left(\sigma Q^{*}\left(\Lambda^{\prime}\left(Q^{*}\right) Q^{*}+\Lambda\left(Q^{*}\right)\right)\left(1-\frac{1}{\sigma}\right)^{-1} \gamma\left(1-\left(\frac{\rho}{\gamma(1+\phi)}\right)^{1-\frac{1}{\sigma}}\left(\frac{1}{\sigma}\right)^{\frac{1}{\sigma}}\right)-(\rho-\gamma)\right)
\end{aligned}
$$

Finally, we can check that $\operatorname{tra} J=\gamma+G_{c}\left(c^{*}, Q^{*}\right)=\rho>0$ and

$\operatorname{det} J=\gamma G_{c}\left(c^{*}, Q^{*}\right)+G_{Q}\left(c^{*}, Q^{*}\right)=\sigma Q^{*}\left(\Lambda^{\prime}\left(Q^{*}\right) Q^{*}+\Lambda\left(Q^{*}\right)\right)\left(1-\frac{1}{\sigma}\right)^{-1} \gamma^{2}\left(1-\left(\frac{\rho}{\gamma(1+\phi)}\right)^{1-\frac{1}{\sigma}}\left(\frac{1}{\sigma}\right)^{\frac{1}{\sigma}}\right)$.

Therefore, $\operatorname{det} J$ is negative: the steady state $\left(c^{*}, Q^{*}\right)$ is saddlepoint stable. 